This item was submitted to Loughborough's Research Repository by the author.

Items in Figshare are protected by copyright, with all rights reserved, unless otherwise indicated.

\title{
A tool for signage placement recommendation in hospitals based on wayfinding metrics
}

\section{PLEASE CITE THE PUBLISHED VERSION}

https://doi.org/10.1177/1420326X17695375

\section{PUBLISHER}

(C) The Author. Published by SAGE Publications (UK and US)

\section{VERSION}

AM (Accepted Manuscript)

\section{PUBLISHER STATEMENT}

This work is made available according to the conditions of the Creative Commons Attribution-NonCommercial 4.0 International (CC BY-NC 4.0) licence. Full details of this licence are available at: http://creativecommons.org/licenses/by-nc/4.0/

\section{LICENCE}

CC BY-NC 4.0

\section{REPOSITORY RECORD}

Greenroyd, Fraser L., Rebecca Hayward, Andrew D.F. Price, Peter Demian, and Shrikant Sharma. 2019. "A Tool for Signage Placement Recommendation in Hospitals Based on Wayfinding Metrics". figshare. https://hdl.handle.net/2134/24184. 


\title{
A tool for signage placement recommendation in hospitals based on wayfinding metrics
}

\author{
Fraser L. Greenroyd*, Rebecca Hayward, Andrew Price, Peter Demian, Shrikant Sharma
}

*Corresponding author - f.greenroyd@lboro.ac.uk

\begin{abstract}
Navigating a healthcare facility can prove challenging to both new and existing patients and visitors. Poor or ineffective use of signage within the facility may enhance navigational difficulties. Signage strategies within facility design tend to be produced without consideration of how people typically navigate a space. Thus, strategies that 'work on paper' may not, in reality, aid or optimize patient and visitor wayfinding. Existing strategies for determining signage placement may also prove costly in terms of time spent on manual analysis of a facility's floor space, including the potential for overlooking prime signage locations when analysing large floor plans. This paper presents a tool which aims to aid signage placement strategies by analysing facility design and routes within it, based on natural wayfinding metrics found in existing literature. The tool is designed to enable quick analysis of large designs for analysing multiple routes, highlighting areas where signage placement would aid natural wayfinding. The outputs of the tool are presented as a colour map which overlays the original 3D model design, highlighting the key areas where signage may be appropriate. An example of how the tool can be utilised to aid effective sign strategy is demonstrated on a small healthcare facility design.
\end{abstract}

\section{Introduction}

Wayfinding as a concept of space legibility originated in $1960^{1}$ and has since grown into a large body of research for navigation around urban settings,,${ }^{2,3}$ airports, ${ }^{4}$ shopping centres ${ }^{5}$ and hospitals.., 7 The act of wayfinding is typically divided into three categories - recreational, resolute and emergency. ${ }^{4}$ In healthcare environments, wayfinding is typically resolute or emergency, whereby patients or visitors are aiming to reach their destination as quickly as possible, either for an appointment, visiting a patient in a ward, or finding the Emergency Department. Hospitals can be difficult places to navigate with a lack of 
visual cues provided by the built environment available, ${ }^{5,8}$ however, environmental clues can be added in the form of architectural differentiation, maps, or signage.

Corridors within healthcare environments link together to form a complex network connecting various aspects of the hospital. There can be little to differentiate between two corridors, causing confusion and frustration among those attempting to navigate them. ${ }^{9}$ One strategy to aid wayfinding involves 'zoning' sections of the hospital, by assigning each department with their own colour and then painting the confines of that department in that colour. This can have an added benefit of making areas attractive and can be used to send patients down paths of the designer's choosing. ${ }^{10-13}$ However, this conflicts with other studies which suggest colours should be kept in neutral or calming tones, such as blue or green, to decrease stress in patients and reduce the chance of delirious episodes caused by abstract colour schemes. ${ }^{14,15}$

Signs and maps are commonly used to aid navigation, though maps are considered to be less effective compared with signage. ${ }^{4,8,16-18}$ Maps can be misinterpreted and can cause further disorientation, rather than aiding the traveller in working out a route to follow. In particular, 'You Are Here' (YAH) maps can prove the most difficult to understand depending on their orientation. For example, if a YAH map is orientated to follow the compass points (i.e. the top of the map corresponds to the north of the building) but the traveller is facing south, the map is effectively upside down and the map may be misread, with one in three people going the wrong way after consulting a YAH map., ${ }^{4,19}$

Maps on their own are used effectively by travellers, however, when signage is available, map usage was found to drop by nearly two-thirds. ${ }^{18}$ This suggests that signage can therefore be a more effective addition to the environment to aid wayfinding, without causing potential health issues associated with a colour 
zoned building and avoiding maps which may disorientate the patient further. Provided the signage is transferring information which is useful to the traveller, journey times can be reduced when compared with those which rely on maps. ${ }^{17}$ There are many recommendations and guidelines in the literature on how to make effective use of signage to aid wayfinding within healthcare environments. Signs should be placed in areas where patients will need them, such as decision points (for example, corridor intersections or atriums). They also need to be easy to understand while the patient is resolutely moving to their destination..$^{5,7,8,19-22}$ However, arguments have been made against signage, suggesting that it should be used sparingly as a last resort to aid wayfinding and containing only the necessary information required to aid the traveller. ${ }^{23}$

Thus while good signage is important, it is vital to ensure it contains only essential information to aid the traveller in their journey. A study of library signage found that travellers in areas which have high connectivity, good visibility and a low complexity in the layout could be hindered by misleading signs and end up going in the wrong direction for their destination. ${ }^{24}$ Therefore, signage usage and placement needs to be carefully considered to avoid further confusing travellers. ${ }^{23}$

A set of guidelines and best practices emerging through research and consultancy has resulted from differing opinions and views on what constitutes good signage. These are aimed at guiding designers on the best placement and use of signage for both use generally and within healthcare environments in the National Health Service (NHS). ${ }^{16,25-29}$ However, beyond high-level recommendations that signage placement "is suitable for all users", "consistent" ${ }^{\prime 6}$ and "not obscured or surrounded by clutter", ${ }^{26}$ there is little available in the way of design tools to aid architects and engineers in effective signage placement. It has been suggested that building designers need to survey a space exhaustively so as to become familiar with the layout in order to develop a signage placement strategy. ${ }^{28}$ Designers will typically mark up the 
spatial design with where signage should be placed borne from their experience in signage consultancy or identifying decision points (critical locations for signage) along pathways between the arrival and departure points of the space. ${ }^{7,8,28}$

Thus, it was the aim of this research to develop a tool that could be used by architects and engineers, especially those working on healthcare designs, to inform and recommend effective signage placement strategies based on available knowledge of how humans navigate spaces. This approach has also added further analysis of signage placement, beyond placement at decision points, to include areas where travellers' natural wayfinding may deviate from the necessary path. The tool is intended to complement designers in understanding a layout and streamlining the signage placement design process in order to develop effective signage placement strategies. This paper details the methodology, development, and academic evaluation of the tool developed to aid signage recommendation studies with the introduction of computational analysis.

\section{Tool development}

It has been shown in a variety of studies that people prefer to travel down a path that expends the least amount of energy or physical effort..$^{2,17,30-33}$ The primarily ideal path people will aim to utilise is contested in the literature however between various path types, such as the shortest in regards to the distance travelled ${ }^{31-33}$ or the straightest path of least angular change..$^{32}$ It has also been argued that the ideal path may be the one of least complexity, where the presence of hills, heavy traffic or other impediments in outdoor navigation may render a longer path to be preferred over the shortest. ${ }^{2}$ Similarly, it has been found that individual differences between people, such as gender, or spatial awareness, may impact on the 
wayfinding ability of a person and result in different path types being preferred, such as the path which infers the greatest feeling of safety in the traveller, or one which is most familiar, preferred over the shortest. ${ }^{2,19}$ Knowing how people navigate through unfamiliar spaces is key to determining their likely path if left without signage in an unfamiliar healthcare environment.

Of the three common types of wayfinding, it is unlikely that people would navigate a hospital for recreational gain. Wayfinding in this category is typically undertaken by tourists on an urban scale, where they might venture around a city and are open to detours and distractions where interesting sights or activities may be found. However, in healthcare settings it is more likely that travellers are wayfinding in the resolute or emergency categories. Wayfinding in the resolute category is typically done when there is a fixed destination and a time to arrive by, such as a patient attending an outpatient appointment. Wayfinding in the emergency category is typically done when there is a shortage of time to reach the destination.

As a traveller moves throughout a space, be that an urban setting or an internal building such as a hospital or an airport, the mode in which the traveller wayfinds may evolve during the journey. It may begin in a resolute mode when the patient moves towards their designated department, which in turn may evolve into the emergency category as the appointment time gets closer.

It is therefore apparent that the ideal path when navigating healthcare environments is one which allows the patient to get to their destination in the shortest time. However, when navigating an unfamiliar location it is unlikely that a patient would have such knowledge of the facility to be able to work out the shortest route without guidance. When faced with navigating unfamiliar locations travellers rely on their 
natural wayfinding skills to find their way around. These skills, typically learnt as children, ${ }^{34,35}$ vary from person to person depending on their gender, background and spatial awareness. ${ }^{19}$

The natural skill of wayfinding is made up of a four step process: orientation; route selection; route control (constant confirmation that the traveller is following their selected route); and recognition of the destination. ${ }^{8,36}$ Route selection can be influenced and aided by a number of interventions, including signage, maps and previous experience of the space. However, if these do not exist, route selection may comprise a step-by-step process using clues from the environment or by following a natural path.

The natural path may be a combination of several spatial navigation techniques and will serve to satisfy the traveller's other psychological needs, such as the need to not feel lost. ${ }^{37}$ This can include a natural decision on which way to turn when faced with a T-Junction. Without intervention from the environment, it has been suggested that travellers will turn to the right at a T-Junction, however it has been argued that driving habits may influence a traveller's decision to turn left or right at a T-Junction. ${ }^{38,39}$

Other common natural paths include the path with the least angular deviation (i.e. keeping on a straight path), ${ }^{2,30,37,40}$ or the path of least complexity (i.e. least number of turns, avoiding backtracking, or least number of decisions). ${ }^{35,37,41-43}$ Other natural path choices may include the widest option (i.e. following a wider corridor round rather than keeping to the straightest path), ${ }^{38}$ or the most visibly accessible option. $5,7,44$

Other metrics which are used to measure a spatial layout's ability to aid natural wayfinding and natural path-taking, include those measuring spatial connectivity and centrality, ${ }^{40,42,44-46}$ however, these measure the space or network graph as a whole. For example, the measure of betweenness centrality ${ }^{46,47}$ identifies 
how central a node in a network graph is, based on how often is it traversed in the shortest paths connecting other nodes. This requires knowledge of the graph as a whole, including all of the shortest paths between nodes and how often that particular node is traversed in those paths. However, when travelling through a space for the first time, humans are unable to know how well connected the next node is based on these metrics. As such, these analysis metrics may not be best suited for the analysis of signage placement within a healthcare facility.

Rather, it may be best to compare the ideal path against the natural path and isolate the areas along the ideal path where the natural path is different. A tool which can analyse several routes, comparing the ideal path to the natural path(s), allows designers, architects and signage consultants to develop effective signage strategies efficiently. Comparing the ideal path to the natural path(s) allows for signage strategies that directly align with a patient's travelling path, aiding the patient in reaching their destination effectively.

Figure 1 displays the typical process a designer might take using this tool. Routes' start and end points are identified by the designer, from which the tool calculates the ideal path for each route. The decision points along each route are identified by analysing the number of direct connections to other nodes. Following this, an analysis against the natural path may be conducted. The natural paths selected for inclusion in the tool following a review of the literature are shown in Table 1. 


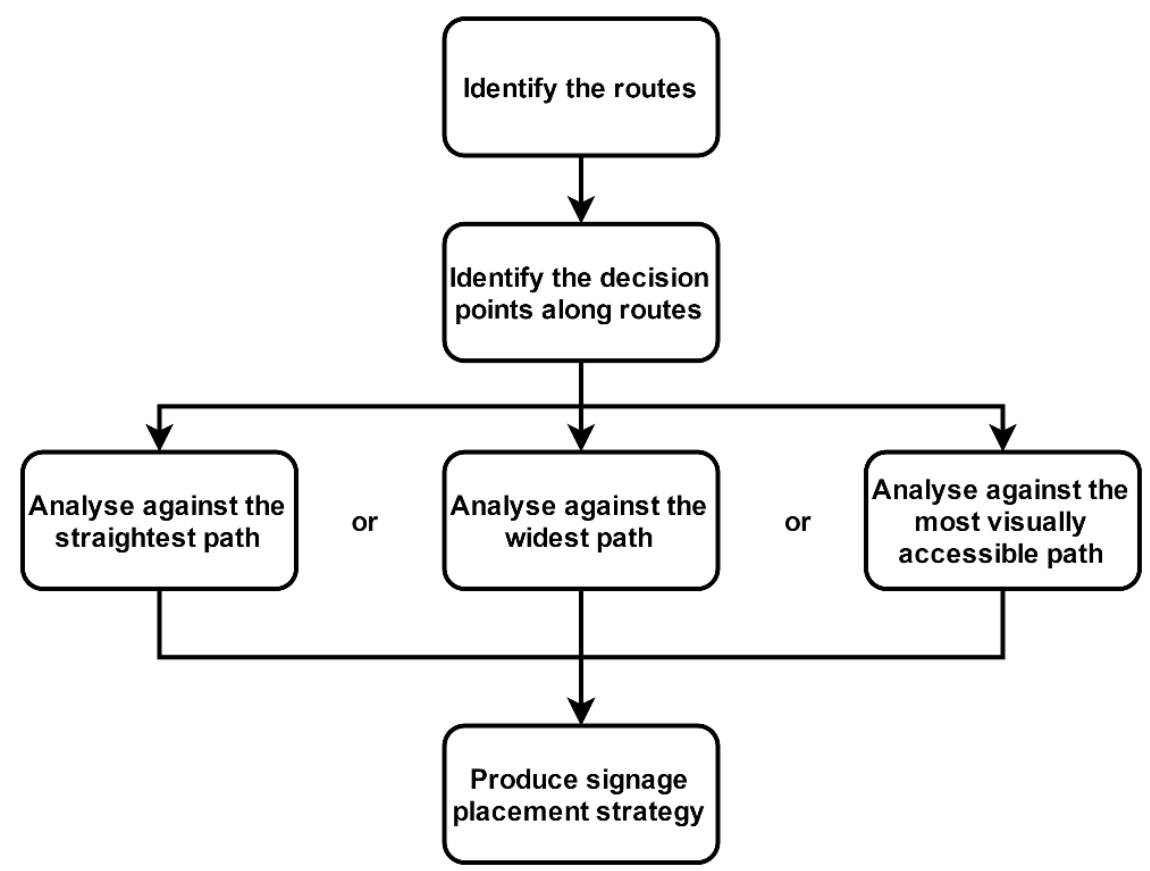

Figure 1 - Flowchart of using the research tool

Table 1 - Natural paths included in the research tool

\begin{tabular}{|l|l|}
\hline \multicolumn{1}{|c|}{ Path } & \\
\hline Straightest & Travellers prefer to continue on the path of least angular deviation \\
\hline Widest & Travellers prefer to continue on the widest path (i.e. the widest corridor will be the \\
& preferred option at an intersection)
\end{tabular}


The analysis between the natural path and the ideal path is conducted on a spatial drawing that may be typically provided by architects during a building's design or assessment of an existing facility. The tool divides the spatial design into a collection of nodes using a combination of Voronoi diagram techniques and graph theory. A Voronoi diagram divides a given space into a number of regions from a given number of points (seeds), with each region containing all of the points in the space which are closer to one seed than to another. ${ }^{48}$ Graph theory is then used to connect these regions by their centre points to produce a 1D navigational network graph of the space. An example of this space division technique is used in the people flow simulator, SmartMove, ${ }^{49,50}$ which uses the points denoting the wall edges in the drawing to begin the Voronoi technique. These nodes contain links to each other generating a 1D network but are still contained within the building model, allowing spatial information such as connection widths and distances to be accurately maintained for analysis. These links to the building model allow the network to retain both $2 \mathrm{D}$ information of the space and $3 \mathrm{D}$ information, primarily where connections between the $\mathrm{Z}$ dimensions occur (such as stairs), allowing for the analysis of paths that traverse multiple floors of a hospital.

The ideal (shortest) path between two given points is calculated using the $\mathrm{A} *$ search algorithm and stored as a collection of the nodes which the ideal path traverses, in the order it traverses them. Following this calculation, the tool begins to calculate what the natural path is and where this deviates from the ideal path. This is achieved with the assumption that the traveller has knowledge of their immediate next step (i.e. they begin on node one and they know how to move to node two initially). This assumption is made to provide an initial direction of travel. From there, the chosen metric is applied to identify what the next node is in the natural path. This node is compared with the next node in the ideal path and if the two nodes are different, the current node is given a flag to indicate that, at that position, it is possible for the natural path to deviate from the preferred and so some intervention may be of use here to guide the 
traveller down the ideal path. The node which conforms to the natural path is also given a flag to highlight which way the natural path might draw the traveller. If the two nodes are the same, then no flag is applied to either node and the tool moves on to calculating the next node.

The pseudo code in Algorithm 1 shows the principle of how nodes are calculated as needing signage or not, while the pseudo code in Algorithm 2 shows the principle of how the next node is calculated during the analysis of the natural path.
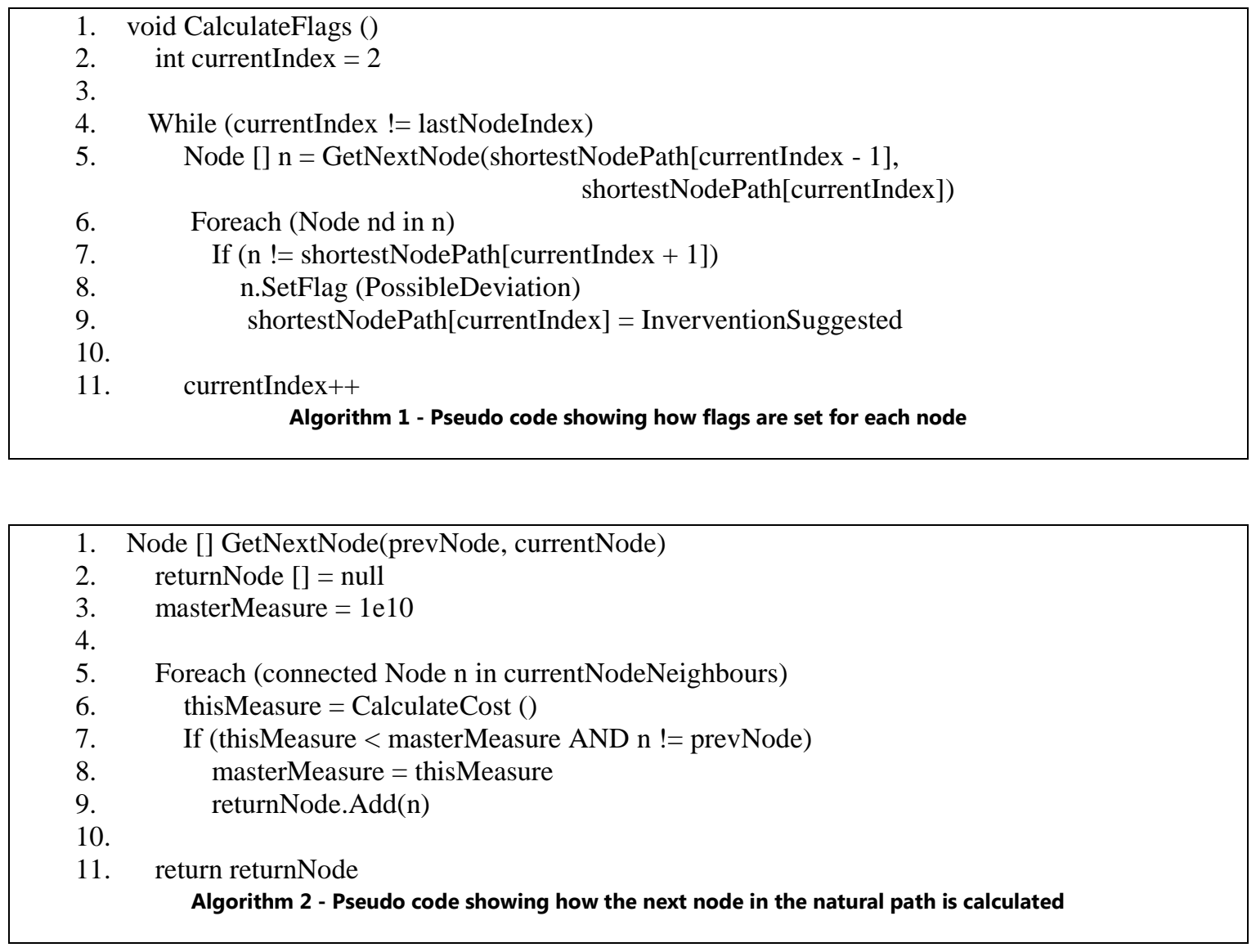
Each metric calculates the cost of each of the connected node's neighbouring nodes to find the one which conforms greatest to the natural path. The cost is calculated so that the most favourable node will have the lowest cost and it is the most favourable node which is checked against the next node in the ideal path.

\section{Analysis algorithms}

The algorithms which were implemented in measuring the natural path were adopted from metrics found in literature, or implemented in other spatial analysis tools where the algorithms have undergone a peer review process or been adopted by a variety of tools in industry and academia. For the purpose of clarity, the implementation of each analysis metric is provided here.

\section{Decision points}

The decision-points algorithm analyses the ideal path and highlights where travellers may encounter confusion along this path based on the complexity. The algorithm counts the total number of connected nodes to the current node, with a higher number of connected neighbours representing a higher complexity.

Research has shown that travellers wish to avoid a path which requires them to back-track ${ }^{23,41}$. To account for this, the algorithm removes one choice from the complexity measure calculated at each node, to remove the choice of backtracking. This is demonstrated in Figure 2. Therefore, a node with only two connected neighbours will be given a complexity rating of one, identifying the node as having low complexity, whereas a node with five connected neighbours will be given a complexity rating of four, thus identifying a significantly more complex node for the traveller.

Natural Path Type 1 - Visual Accessibility 
Visual accessibility analyses the path with the greatest visibility from the current node. A visible neighbour is defined as any node which the node being analysed has line of sight to, without being impeded by spatial obstacles such as walls or columns, as defined in the geometric spatial design. Of the visible neighbours for a connected node, only those which are also visible from the current node are included in the visual accessibility score for that connected node. For example, if a connected node has line of sight to five other nodes, but only two of them are also visible from the current node, then the visual accessibility score is two which represents the number of visually accessible nodes from the node where the traveller is currently stood, as demonstrated in Figure 3.

Previous research has found that a greater number of visible neighbours assists spatial orientation and wayfinding in travellers ${ }^{24}$, so a node with good visibility may be favoured over others. As such, the visual accessibility analysis looks at the connected node's visibility score and returns the node with the highest visibility. This highlights where travellers may be drawn to areas with higher visibility and away from the ideal path.

\section{Natural Path Type 2 - Straightest Path Calculations}

The algorithm for calculating the straightest natural path is performed by calculating the angle of deviation between the straight line path travelling from the previous node to the current node, and the path taken from the current node to the next node, as demonstrated in Figure 4. The node with the smallest deviation from the straight line is considered to be the most favourable.

\section{Natural Path Type 3 - Width}

Width analysis is based on the principle that travellers have a preference for the widest route option available. The analysis compares the spatial dimensions of connected nodes (not including the previous 
node) to find the node with the widest connection, demonstrated in Figure 5. This node is returned as the most favourable node for the natural path.

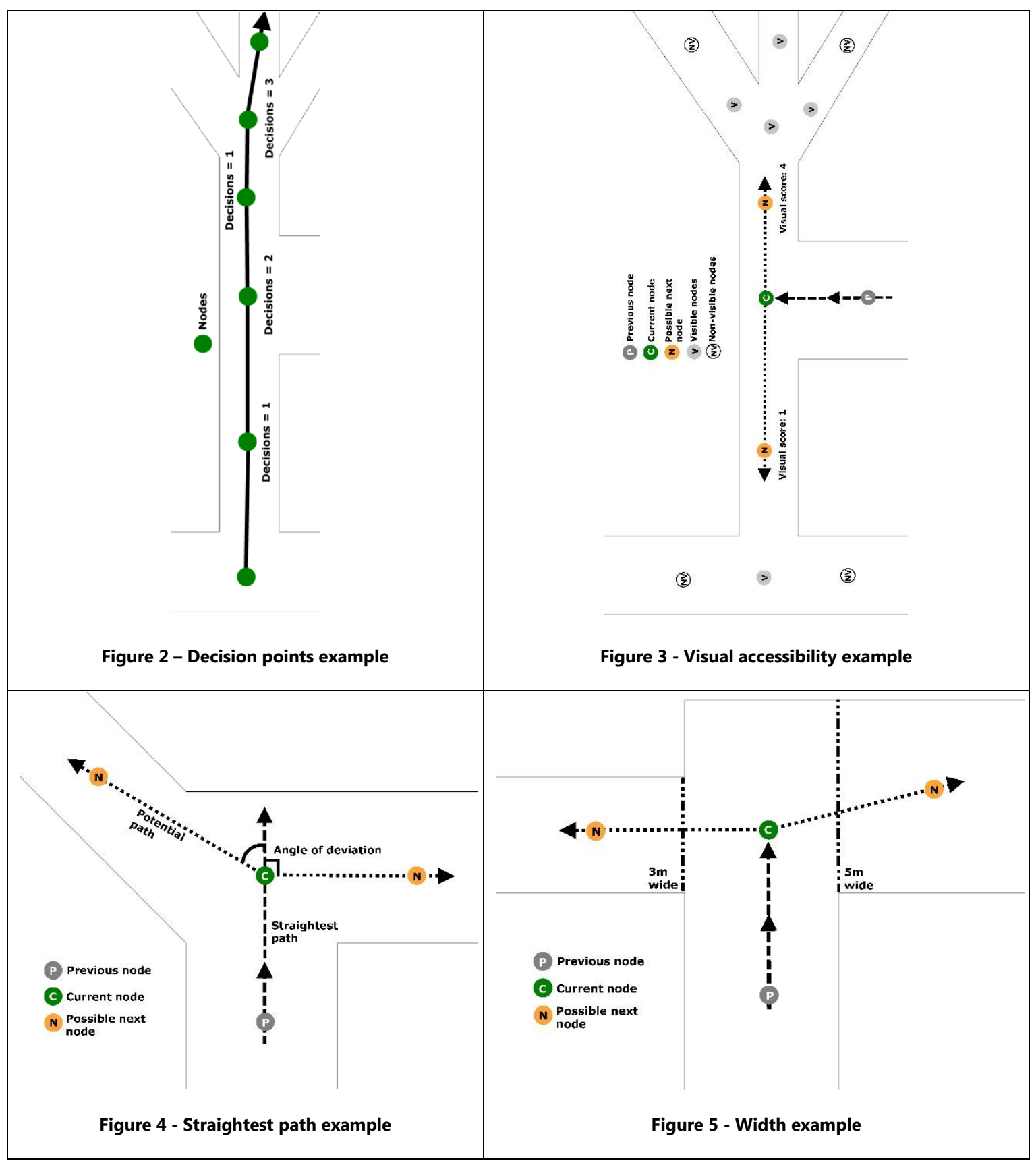




\section{Nodes of equal choice}

It may be possible for the analysis metrics to identify two nodes of equal favourability for the natural path. An example of this may be a T-junction, where both options have a 90 degree angle of deviation, meaning either one may be chosen on the natural path. Where this occurs the tool shows this as an area where an intervention may be beneficial. This is done regardless of whether one of the favourable nodes includes the next node in the ideal path, as there is still the potential for travellers to take the wrong path without intervention.

\section{Visualisation of results}

Visualisation plays an important role in the ability to disseminate information between architects, engineers and clients. If the results of the analysis cannot be successfully communicated to stakeholders then they will be unable to act upon the information generated by the tool. This may result in poor signage strategies due to misinterpreted results or confusion over the output and its meaning. Therefore, careful consideration should be given to which visualisation technique makes the most sense to the enduser and associated audiences.

As the key output of the analysis tool is a set of recommendations on where a designer should consider signage placement for the maximum benefit of a traveller (e.g. patient, visitor, etc.). The visualisation output of this tool is designed to show the analysis results in a colour scale ranging from good (areas where intervention is not required) to bad (areas where intervention is required, i.e. areas where travellers start to deviate from the ideal path). Areas where the natural path continues away from the ideal path are shown in a middle colour on the scale - this provides consultants and engineers with knowledge of the natural direction travellers may be drawn towards and may aid in the orientation of signage. 


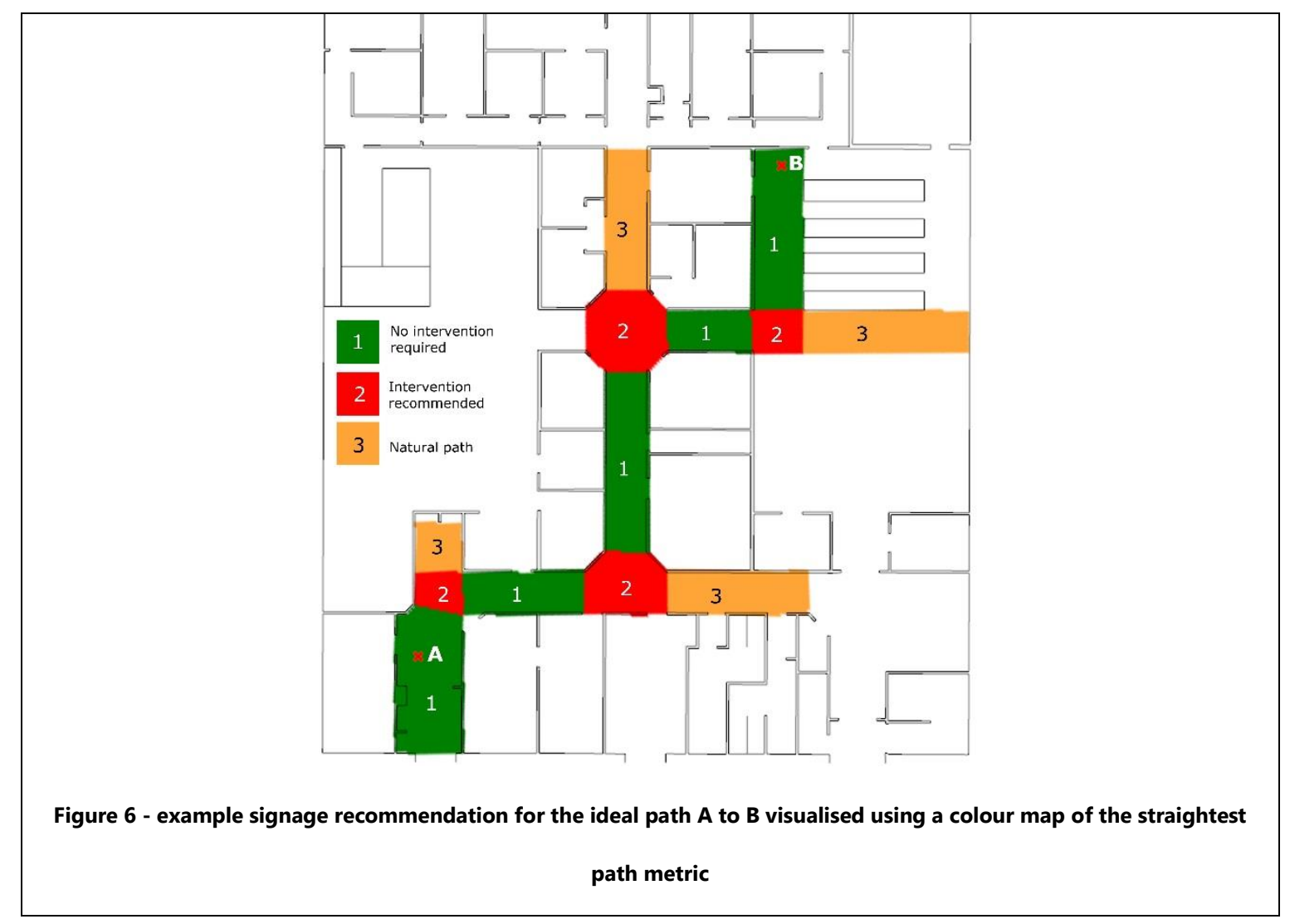

Figure 6 shows the visualisation overlaid onto a CAD drawing that may be typically used by architects or engineers. The output shows the result of the analysis for the chosen metric (in this example the straightest path metric) and highlights where signage would be required to guide users onto the ideal path. Areas highlighted in red are areas where travellers would deviate if they were navigating based on the natural (straightest) path. Signage would therefore be recommended at these points to guide travellers in the correct direction to their destination (point B) on the ideal (shortest) path.

Tool verification Testing of the tool was conducted on a healthcare model from a recent industrial project. This model was a stand-alone pharmacy department (see Figure 7). Each algorithm was tested on the model and compared 
the outputs with those from manual analysis. For the route definition, two points were chosen in the model and the same route was used for each algorithm test. The first point in the route was placed in the entrance area, while the second point was placed towards the rear of the department.

Verification of the tool came from a comparison of manual analysis of the model, performed as if producing a real signage strategy, with the analysis results of the tool. This included measuring the spatial dimensions of the model to ensure these were being taken into account by the tool. The results of this testing are summarised here. For the purposes of this discussion, Model-P is the name given to the test model. Figures 8 to 15 show the results of these tests.

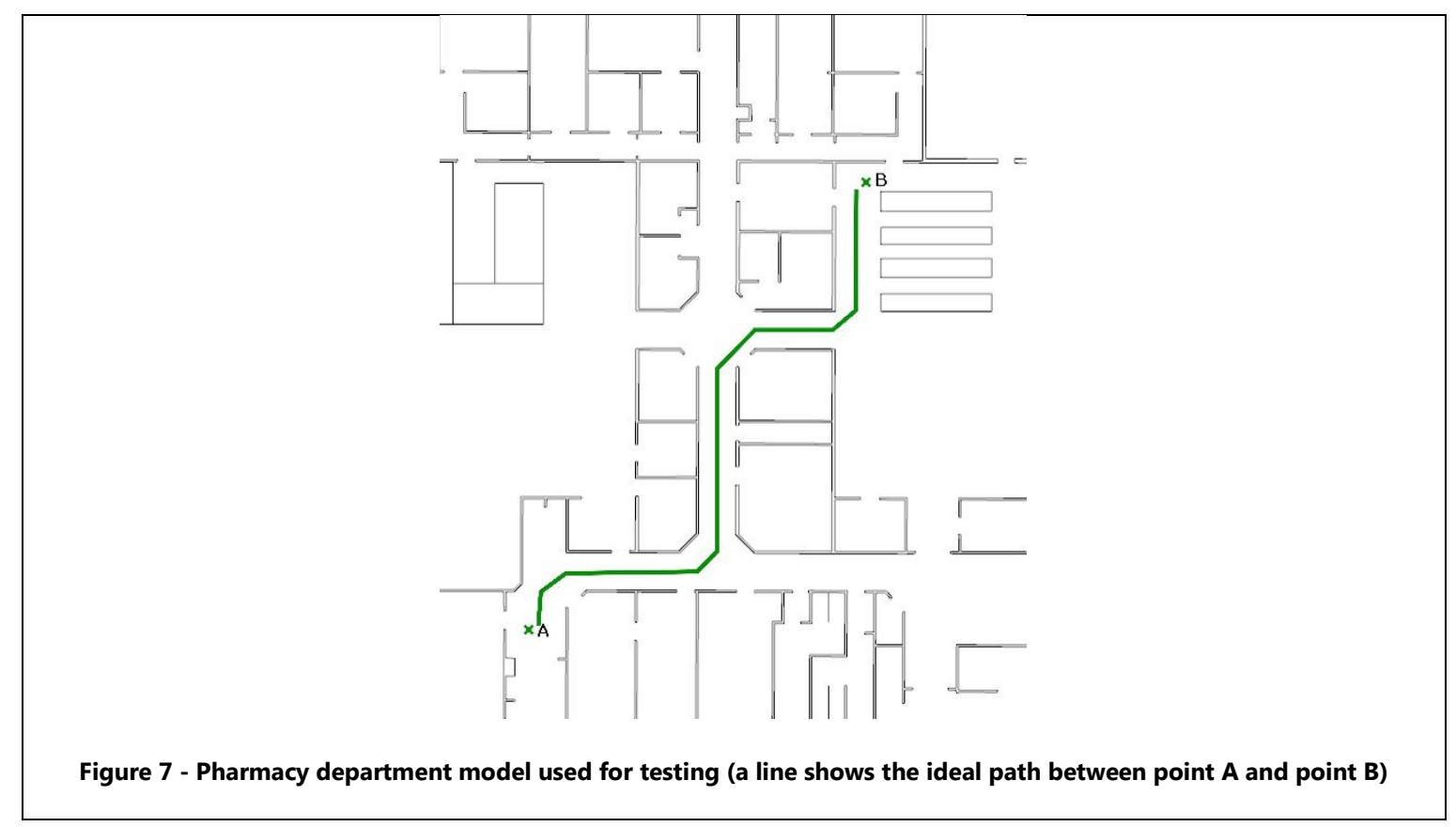



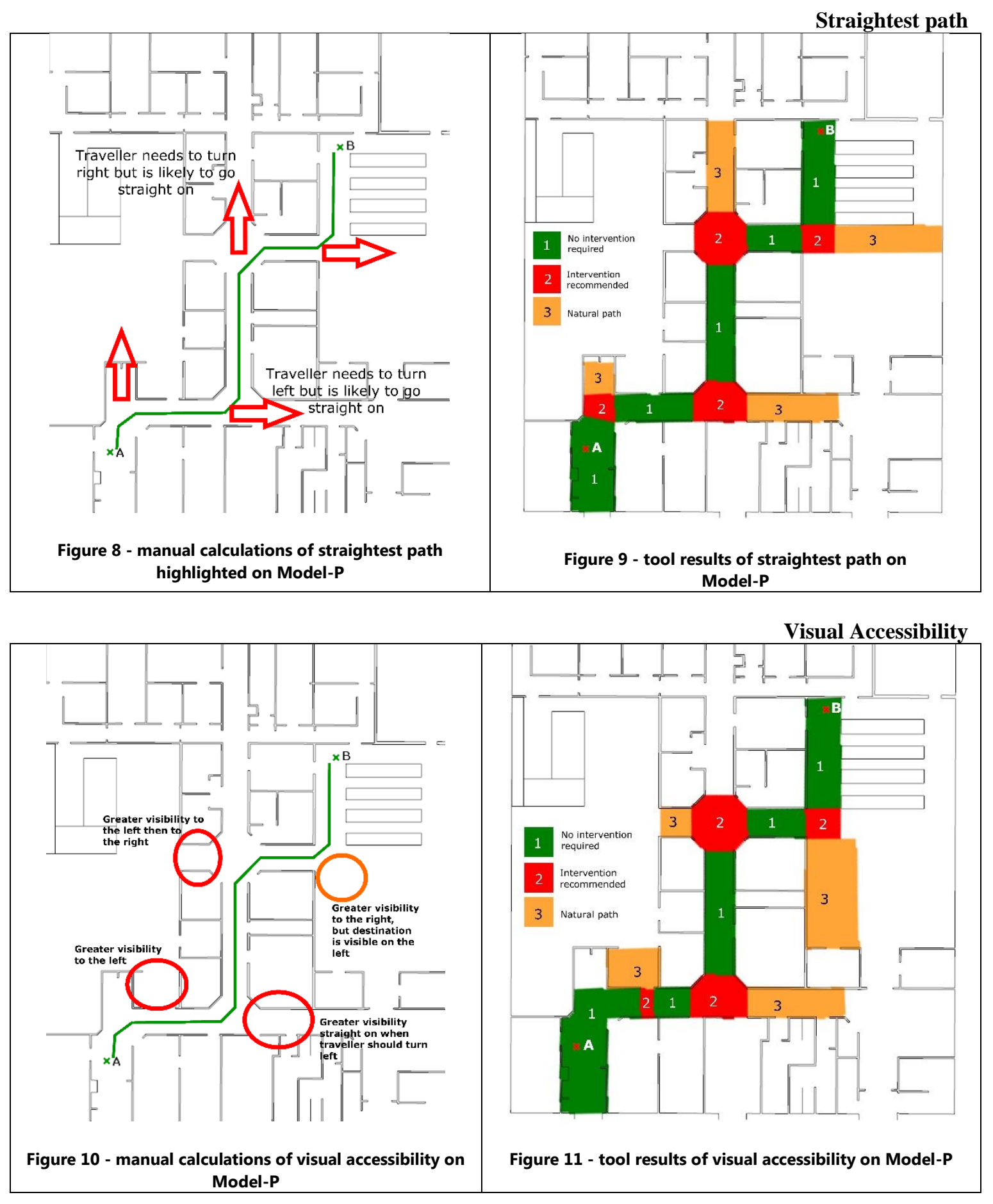

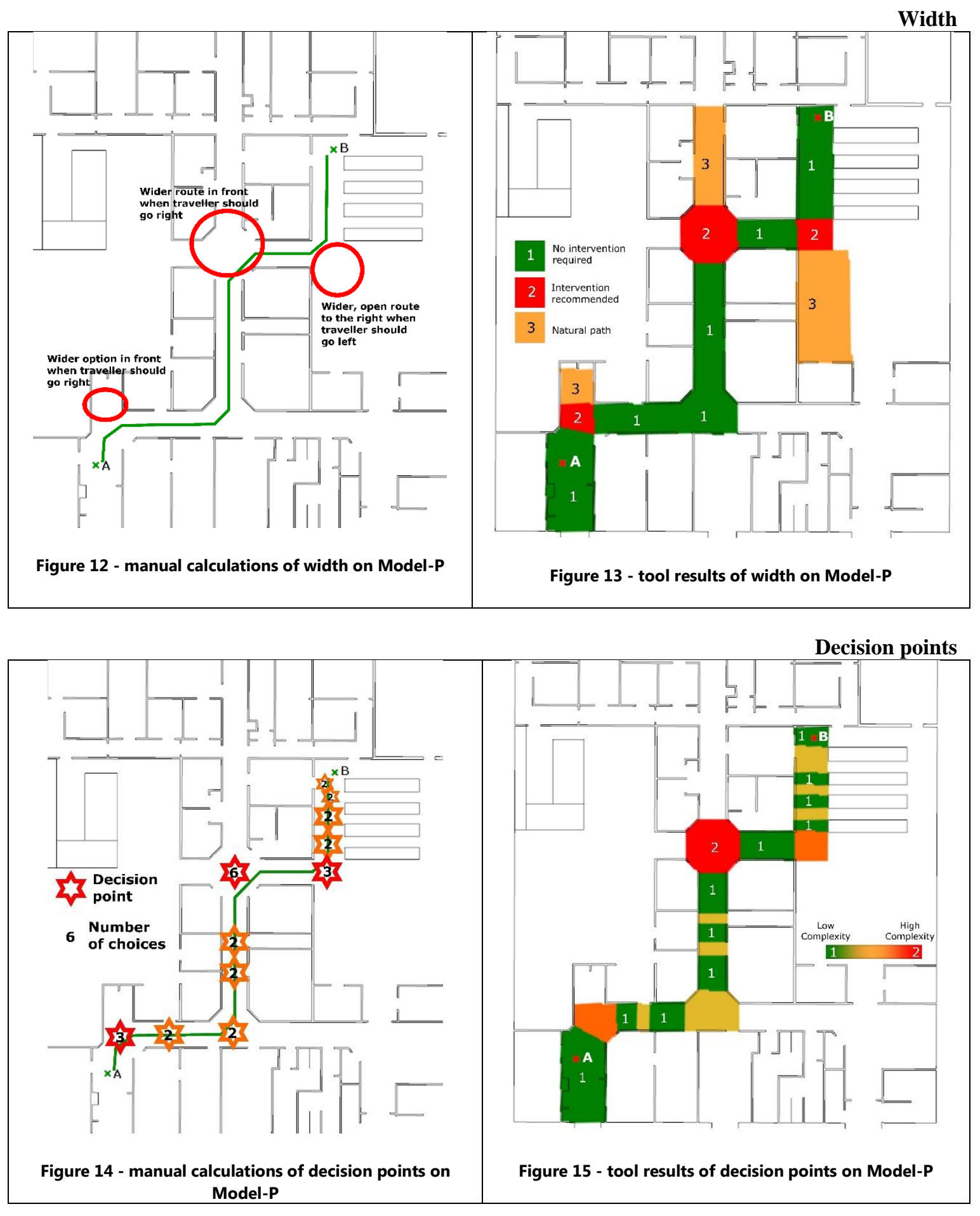


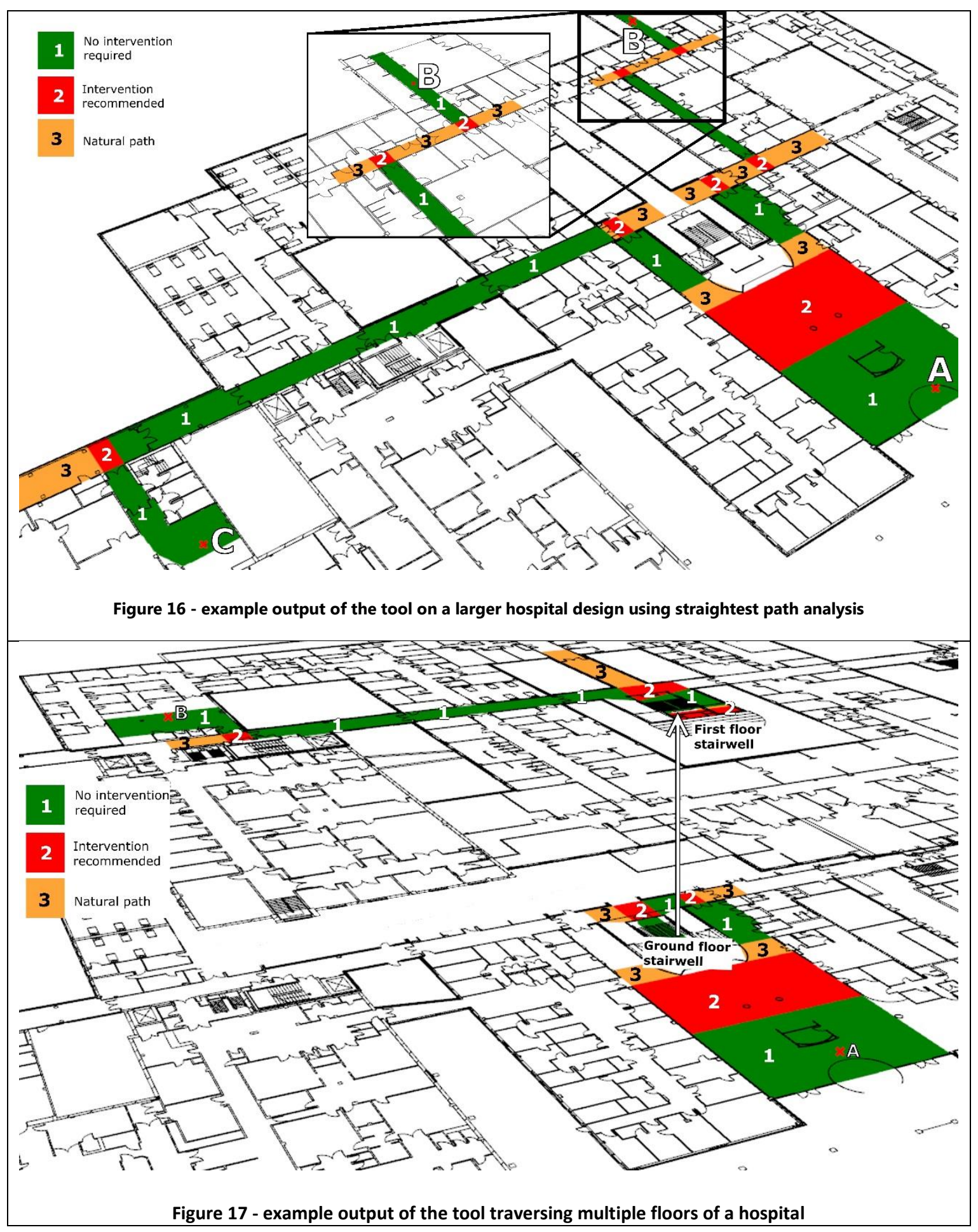




\section{Discussion}

The implementation of these algorithms provides a tool for quick signage recommendation analysis in healthcare facilities. The tool can be easily used by architects, engineers, consultants, and planners to analyse a spatial design and produce a signage strategy that aims to keep patients and visitors on the ideal path to their destinations. Figure 16 shows the use of the straightest path metric being used on a larger hospital model on two routes. This analysed the shortest route between point A and point B, and between point $\mathrm{A}$ and point $\mathrm{C}$. This highlights in red the areas where signage would be best placed to direct travellers to their destination on the ideal path. Figure 17 shows the tool being used to analyse the route between point $\mathrm{A}$ and point $\mathrm{B}$, using the straightest path metric, across multiple floors. On the decisions metric, a change in vertical elevation would be considered by the tool to be an additional decision and this would be highlighted to the engineer as such.

However, the tool should not be used by a user who possesses no wayfinding and signage experience to produce signage strategies, though it may be used as a training tool for those with no experience. Rather, it should be used as a guidance and recommendation tool combined with experience to make a good decision for the needs of the building. The signage recommendation analysis tool provides an analysis of the building being designed and the routes which people may make. This may typically be between the entrance areas and specific hospital departments. These routes are analysed with the aim of keeping the patient on the ideal path and highlights where the natural path (without intervention) may deviate from the ideal path.

The ability of this tool to analyse multiple routes without the need of an engineer exhaustively surveying the space and performing the analysis manually is of great benefit to how signage strategies are produced. The tool can be set up with relative ease, perform the analysis, and produce the output for the engineer to 
examine. This approach enables an efficient signage strategy creation by automating the analysis tasks and allowing a computer to perform them relatively quickly, while retaining the use of human judgement and expertise for the final strategy. The final decision on the placement of signage should always be made by an experienced engineer or consultant, who may know more of the space use than is provided to the tool by the CAD drawing. Additional benefits of this tool can be seen where the building design may be in a state of flux. With manual analysis, an engineer may have to reanalyse an entire model to produce a new signage strategy. Using this tool, the engineer need only update the model and the tool will produce new recommendations in a more time efficient manner.

The benefits of this tool can be seen on large scale plans where manually analysing the space could take a long time, and the possibility exists that an area of the floor plan may be missed through human error. By providing analysis results in an eye-catching warning colour, the engineer/consultant can produce a signage strategy based on these recommendations efficiently.

\section{Limitations}

In the implementation of the tool provided by this research, signage recommendation analysis is performed on one metric at a time as selected by the modeller. If the modeller wishes to analyse a design based on multiple metrics, then the modeller would need to run each analysis separately and then interpret between the outputs for the most optimal signage strategy. This is a deliberate design decision, owing to the lack of literature specifying how the metrics could be meaningfully combined and weighted (i.e. there is little knowledge on how humans navigate on the straightest path and most visually accessible path combined and which factor would take precedence is unknown). If further research was to be conducted and a meaningful hierarchy for multiple metrics established, the tool presented in this paper could be extended to accommodate this. 
At present, the tool analyses the ideal path (the shortest path between two given points) against the chosen metric to produce the signage recommendation. However, there are other path types which may constitute the ideal path in addition to the shortest depending on the type of wayfinding a traveller is undertaking. In this research, the shortest path was used as the ideal path because it best matched with the resolute or emergency types of wayfinding typically found in hospitals. Further development of this tool may find it appropriate to include other path types for the ideal path for using the tool on projects in other sectors.

Some research has suggested that, when faced with an equal choice, travellers will opt to turn right. ${ }^{38,39}$ This could be implemented within a signage recommendation tool, however, it is simpler, and more robust, to highlight areas with multiple choices of equal weighting as an area for potential confusion and offer the recommendation that signage be placed there to counter this, as this tool does.

There may be other metrics which could also be included within the analysis functionality of this tool, such as those found within spatial network topology theory. ${ }^{40,42,44,45}$ There are measures of spatial connectivity and centrality which have not been included in this tool that could have a place within the functionality at a later stage. The option not to include these additional metrics and spatial analysis in this signage recommendation tool was based on how travellers actively choose to navigate through a space. For example, some of the centrality metrics work based on knowledge of an entire model. However, when travelling through a space for the first time, humans are unlikely to know how central a certain area is compared with another. If further research offered tangible evidence that humans do navigate using these metrics (either consciously or subconsciously) then the tool could be extended accordingly to include them. 
Guidelines for signage placement recommend that the distance between signage in corridors should not exceed 30 meters. ${ }^{16}$ Repeater signs are used to give travellers confirmation they are still heading in the correct direction. This particular signage recommendation factor is not currently implemented within the tool, as confidence in a route is not a factor which is considered. However, future research and development of the tool may see fit to include this measure to provide further signage recommendation strategies.

Finally, the tool presented here is used as a 'static' analysis tool, used on designs and drawings prior to construction or renovation. As such, the tool does not take into account the impact of crowd movements or other pedestrian flows on the path travellers may take when moving through a hospital. However, the tool could be adapted to take these into account in further research, allowing for signage recommendations to be made based on the natural path taking into account the impact of other traveller movements.

\section{Conclusion}

This research has developed a tool to aid signage placement. This tool allows engineers, architects and healthcare planners to analyse multiple routes through a spatial design and produce an optimal signage strategy based on keeping patients and visitors on the ideal path. This is achieved by analysing the natural path and highlighting where the natural path is likely to deviate from the ideal path. The tool combines the benefits of computational working - quick processing and analysis - with the benefits of human judgement and expertise. This tool can be used to analyse multiple routes across any spatial design efficiently and without missing routes, saving an engineering team from having to exhaustively study the design manually. 
At the time of writing up this research, the tool has begun to enter parallel implementation within an industrial company, being used in combination with existing manual methods to analyse wayfinding and signage issues. Early anecdotal evidence from willing adopters suggests the tool is beneficial to the workings of the users, however, use is still in its infancy. Once the tool has been used for a period of time, to allow users to get used to working with it and to account for any potential "honeymoon effect" caused by the introduction of the tool, a full evaluation study would be beneficial to understand whether the tool is having the predicted benefits on signage recommendation work-flow. It is hoped that this tool will aid signage strategists to produce effective signage placement strategies efficiently, allowing more time to be spent on refining the signage itself, or refining other aspects of the healthcare design project.

\section{Acknowledgement}

We would like to thank the Engineering and Physical Sciences Research Council, and Centre for Innovative and Collaborative Construction Engineering at Loughborough University for provision of a grant (number EPG037272) to undertake this research project in collaboration with BuroHappold Engineering Ltd.

\section{Authors' contributions}

Greenroyd F developed the tool presented in this paper, the literature search and the drafting of this paper. Hayward B and Sharma S contributed to the industrial design and relevance of the project and drafting of this paper. Price A and Demian P contributed to the academic design and relevance of the project and drafting of this paper.

\section{Declaration}

The Author(s) declare(s) that there is no conflict of interest. 


\section{References}

1. Lynch K. The Image of the City. Vol. 11. Cambridge, MA: MIT Press; 1960.

2. Montello DR. The measurement of cognitive distance: Methods and construct validity. J Environ Psychol. 1991;11(2):101-22.

3. Gluck M. Making Sense of Human Wayfinding: Review of Cognitive and Linguistic Knowledge for Personal Navigation with a New Research Direction. In: Cognitive and Linguistic Aspects of Geographic Space. Heidelberg: Springer Netherlands; 1991. p. 117-35.

4. Fewings R. Wayfinding and Airport Terminal Design. J Navig. 2001;54(2):177-84.

5. Dogu U, Erkip F. Spatial Factors Affecting Wayfinding and Orientation: A Case Study in a Shopping Mall. Environ Behav. 2000;32(6):731-55.

6. Cooper R. Successful signage: how hospitals have solved wayfinding challenges. Health facilities management. 2010;23:27-30.

7. Baskaya A, Wilson C, Özcan YZ. Wayfinding in an Unfamiliar Environment: Different Spatial Settings of Two Polyclinics. Environ Behav. 2004;36(6):839-67.

8. Farr AC, Kleinschmidt T, Yarlagadda P, Mengersen K. Wayfinding: A simple concept, a complex process. Transp Rev. 2012;32(6):715-43.

9. Wright P, Hull AJ, Lickorish A. Navigating in A Hospital Outpatients Department - the Merits of Maps and Wall Signs. J Archit Plann Res. 1993;10(1):76-89.

10. Dalke H, Little J, Niemann E, Camgoz N, Steadman G, Hill S, et al. Colour and lighting in hospital design. Opt Laser Technol. 2006;38(4):343-65.

11. Cooper B, Mohide A, Gilbert S. Testing the use of color in a long-term care setting. Dimens Health Serv. 1989;66(6):22-4.

12. Devlin AS, Arneill AB. Health Care Environments and Patient Outcomes: A Review of the Literature. Environ Behav. 2003;35(5):665-94.

13. Passini R, Rainville C, Marchand N, Joanette Y. Wayfinding and dementia: some research findings and a new look at design. J Archit Plann Res. 1998;15(2):133-51.

14. Thompson D, Hamilton D. Guidelines for intensive care unit design. Crit Care Med. 2012 May;40(5):1586-600.

15. Kiekkas P, Theodorakopoulou G, Spyratos F, Baltopoulos G. Psychological distress and delusional memories after critical care: a literature review. Int Nurs Rev. 2010;53(7):288-96.

16. Apelt R, Crawford J, Hogan DJ. Wayfinding Design Guidelines. Brisbane: CRC for Construction Innovation; 2007.

17. Butler DL, Acquino AL, Hissong A a, Scott $\mathrm{P}$ a. Wayfinding by newcomers in a complex building. Hum Factors J Hum Factors Ergon Soc. 1993;35(1):159-73.

18. Hölscher C, Büchner SJ, Brösamle M, Meilinger T, Strube G. Signs and Maps - Cognitive Economy in the Use of External Aids for Indoor Navigation Introduction. In: Proceedings of the 29th annual cognitive science society. Cognitive Science Society Austin, TX; 2007. p. 377-82. 
19. Montello DR, Sas C. Human Factors of Wayfinding in Navigation. London: CRC Press/Taylor \& Francis Ltd.; 2006.

20. Orellana N. On Spatial Wayfinding: agent and human navigation patterns in virtual and real worlds. University College London; 2012.

21. Rousek JB, Hallbeck MS. Improving and analyzing signage within a healthcare setting. Appl Ergon. 2011;42(6):771-84.

22. Hashim MJ, Alkaabi MSKM, Bharwani S. Interpretation of way-finding healthcare symbols by a multicultural population: Navigation signage design for global health. Appl Ergon. 2014;45(3):503-9.

23. Carr AR. An Experiment with Art Library Users, Signs, and Wayfinding. Chapel Hill, NC Sch Inf Libr Sci Univ North Carolina Chapel Hill. 2006;

24. Li R, Klippel A. Wayfinding in Libraries: Can Problems Be Predicted? J Map Geogr Libr. 2012;8(1):21-38.

25. Bonfanti A. Towards an approach to signage management quality (SMQ). J Serv Mark. 2013;27(4):312-21.

26. Department of Health. Wayfinding - Effective Wayfinding and Signing Systems Guidance for Healthcare Facilities (supersedes HTM 65 “Signs”). The Stationary Office. London; 2005.

27. Buechner SJ, Wiener J, Hölscher C. Methodological triangulation to assess sign placement. In: Proceedings of the Symposium on Eye Tracking Research and Applications - ETRA '12. Santa Barbara; 2012. p. 185-8.

28. Gibson D. The wayfinding handbook: Information design for public places. New York: Princeton Architectural Press; 2009.

29. Calori C, Vanden-Eynden D. Signage and Wayfinding Design: A Complete Guide to Creating Environmental Graphic Design Systems. San Francisco: John Wiley \& Sons; 2015.

30. Fu E, Bravo M, Roskos B. Single-destination navigation in a multiple-destination environment: a new "later-destination attractor" bias in route choice. Mem Cognit. 2015;43(7):1043-55.

31. Lwin KK, Murayama Y. Modelling of urban green space walkability: Eco-friendly walk score calculator. Comput Environ Urban Syst. 2011;35(5):408-20.

32. Peponis J, Bafna S, Zhang Z. The connectivity of streets: Reach and directional distance. Environ Plan B Plan Des. 2008;35(5):881-901.

33. Cutts BB, Darby KJ, Boone CG, Brewis A. City structure, obesity, and environmental justice: An integrated analysis of physical and social barriers to walkable streets and park access. Soc Sci Med. 2009;69(9):1314-22.

34. Piaget J, Inhelder B. The child's conception of space. London: Routledge \& K. Paul; 1967.

35. Raubal M, Egenhofer MJ. Comparing the complexity of wayfinding tasks in built environments. Environ Plan B Plan Des. 1998;25(6):895-914.

36. Downs R, Stea D. Image and Environment: Cognitive Mapping and Spatial Behavior. Chicago: Transaction Publishers; 1973. 79-86 p.

37. Shao J, Kulik L, Tanin E, Guo L. Travel Distance versus Navigation Complexity: A Study on Different Spatial Queries on Road Networks. In: Proceedings of the 23rd ACM International 
Conference on Information and Knowledge Management. Shanghai: ACM; 2014. p. 1791-4.

38. Vilar E, Rebelo F, Noriega P, Duarte E, Mayhorn CB. Effects of competing environmental variables and signage on route-choices in simulated everyday and emergency wayfinding situations. Ergonomics. 2014;57(4):511-24.

39. Scharine A a, McBeath MK. Right-handers and Americans favor turning to the right. Hum Factors Ergon Soc. 2002;44(2):248-56.

40. Mohamad WSNW, Said I. A review of variables of urban street connectivity for spatial connection. IOP Conf Ser Earth Environ Sci. 2014;18(1):12173.

41. Peponis J, Zimring C, Choi YK. Finding the building in wayfinding. Environ Behav. 1990;22(5):555-90.

42. Jiang B, Claramunt C, Klarqvist B. Integration of space syntax into GIS for modelling urban spaces. Int J Appl Earth Obs Geoinf. 2000;2(3):161-71.

43. Arthur P, Passini R. Wayfinding: people, signs and architecture. Toronto: Mcgraw-Hill; 1992.

44. Peponis J, Wineman J, Bafna S, Rashid M, Kim SH. On the generation of linear representations of spatial configuration. Environ Plan B Plan Des. 1998;25:559-76.

45. Jiang B, Claramunt C, Batty M. Geometric accessibility and geographic information: Extending desktop GIS to space syntax. Comput Environ Urban Syst. 1999;23(2):127-46.

46. Crucitti P, Latora V, Porta S. Centrality measures in spatial networks of urban streets. Phys Rev E - Stat Nonlinear, Soft Matter Phys. 2006;73(3):36125.

47. Latora V, Marchiori M. A measure of centrality based on network efficiency. New J Phys. 2007;9(6):188-204.

48. Hoff III KE, Culver T, Keyser J, Lin M, Manocha D. Fast computation of generalized Voronoi diagrams using graphics hardware. In: Proceedings of the 26th annual conference on Computer graphics and interactive techniques. Los Angeles: ACM Press/Addison-Wesley Publishing Co.; 1999. p. 277-86.

49. Varughese JS, Bouchlaghem D, Brocklehurst D, Sharma S. People Flow Modeling in Building Design. In: Computing in Civil and Building Engineering, Proceedings of the International Conference. Nottingham; 2010. p. 59.

50. Sharma S, Fisher A. Simulating the user experience: Design optimisation for visitor comfort. Archit Des. 2013;83(2):62-5. 